\title{
Correlations between visual acuity and macular microvasculature quantified with optical coherence tomography angiography in diabetic macular oedema
}

\author{
Chia-Chieh Hsiao ${ }^{1,2} \cdot$ Chung-May Yang ${ }^{1,3} \cdot$ Chang-Hao Yang ${ }^{1,3} \cdot$ Tzyy-Chang Ho $^{1} \cdot$ Tso-Ting Lai $^{1} \cdot$ Yi-Ting Hsieh $^{1}$
}

Received: 29 October 2018 / Revised: 13 May 2019 / Accepted: 29 June 2019 / Published online: 12 August 2019

(c) The Author(s), under exclusive licence to The Royal College of Ophthalmologists 2019

\begin{abstract}
Purpose To explore the impact of macular ischaemia on vision in diabetic macular oedema (DMO) by analysing the correlations between visual acuity and macular microvascular parameters using optical coherence tomography angiography (OCTA).

Methods OCTA was performed in 81 eyes of 48 patients with DMO, and $3 \times 3-\mathrm{mm}^{2}$ en face OCTA images of the superficial capillary plexus and deep capillary plexus in the central macula were retrospectively collected. Microvascular parameters including the number of microaneurysms, area of foveal avascular zone (FAZ), acircularity index of FAZ, vessel density, skeleton density, vessel density index and fractal dimension were measured. Central retinal thickness (CRT) and the presence of ellipsoid zone disruption at the fovea were also recorded. Linear mixed models were used to evaluate the correlations between best-corrected visual acuity (BCVA) and the microvascular parameters.

Results After adjustment for CRT and ellipsoid zone disruption at the fovea, lower skeleton density and lower fractal dimension in the deep capillary plexus were correlated with poorer BCVA $(P=0.030$ and 0.024 , respectively). None of the microvascular parameters of the superficial capillary plexus were correlated with BCVA after adjustment for CRT and ellipsoid zone disruption (all, $P>0.05$ ).

Conclusions For eyes with DMO, low skeleton density and low branching complexity in the deep capillary plexus of central macula were correlated with poor vision. OCTA could offer quantified parameters of macular microvasculature to measure the impact of macular ischaemia on visual acuity in DMO.
\end{abstract}

\section{Introduction}

Diabetic retinopathy (DR) is a leading cause of blindness among the working-age population, affecting 93 million persons worldwide. Of them, up to 21 million persons have diabetic macular oedema (DMO), which may cause severe visual impairment [1]. Retinal ischaemia in DR leads to the release of vascular endothelial growth factor (VEGF), breakdown of the blood-retinal barrier, increased

Yi-Ting Hsieh

ythyth@gmail.com

1 Department of Ophthalmology, National Taiwan University Hospital, Taipei, Taiwan

2 Department of Ophthalmology, En Chu Kong Hospital, New Taipei, Taiwan

3 College of Medicine, National Taiwan University, Taipei, Taiwan permeability of macular retinal vessels, and exudation of serous fluid and lipids into the macula, resulting in macular oedema and vision deterioration [2, 3]. Macular ischaemia may also occur in patients with DMO, which may cause further deterioration of vision. However, the relationship between visual acuity and the extent of macular ischaemia in patients with DMO is still unclear.

Optical coherence tomography angiography (OCTA) is a recently developed technique which can visualise the retinal vascular structure [4-7]. Compared with the traditional fluorescein angiography (FA), OCTA allows the observation of blood flow in each retinal capillary layer without dye injection. The AngioVue system (Avanti OCT; Optovue, Fremont, CA, USA) uses a split-spectrum amplitudedecorrelation angiography (SSADA) algorithm, and can divide the retina-choroid layer into four layers: the superficial capillary plexus (SCP), deep capillary plexus (DCP), outer retina and choroidal capillary layer [8, 9]. It can clearly demonstrate the microvascular changes in diabetic 
retinopathy, such as distortion of the foveal avascular zone, retinal capillary dropout and pruning of arteriolar branches [10]. Several parameters in OCTA have been proposed to represent the microvascular structure and perfusion status in the macula. Fractal dimension is a ratio that provides a statistical index of complexity, and it has been used for measuring the vasculature branching complexity and characterising the growth of neurons, tissues and vessels $[11,12]$. Vessel density (which represents the proportion of area occupied by vessels), vessel skeleton density (which represents the density of vascular architecture itself) and vessel diameter index (which represents the average vessel calibre) are also OCTA parameters for quantitative analysis of retinal microvasculature [13]. Microvasculature changes with progressive vessel density loss and morphological changes with decreasing fractal dimension have been demonstrated as DR worsens $[14,15]$. A recent study also showed the area of the foveal avascular zone (FAZ) was significantly correlated with visual acuity in DR patients [16]. The aim of this study was to explore the impact of macular ischaemia on the vision of patients with DMO by analysing the associations between visual acuity and retinal microvascular density, as well as morphological characteristics using OCTA.

\section{Patients and methods}

\section{Study population}

The data of type 2 diabetes patients who had been diagnosed as DMO at National Taiwan University Hospital between August 2015 and January 2017 were retrospectively collected. The diagnostic criteria for DMO were (1) DR documented by fundus photography or fluorescein angiography (FA); and (2) macular oedema with the presence of retinal thickening, intraretinal cysts, intraretinal hyperreflective foci or subretinal fluid as documented by optical coherence tomography (OCT). The records of eyes examination with RTVue XR OCT Avanti System using AngioVue Version 201.2.0.93 (Optovue, Fremont, CA, USA) and the concurrent records of best-corrected visual acuity (BCVA) measured by Snellen charts were examined. For patients who had received multiple OCTA examinations during the study period, only the earliest one and the concurrent BCVA were used for analysis. Exclusion criteria included (1) eyes with vitreomacular traction or tractional retinal detachment demonstrated by OCT; (2) eyes with choroidal neovascularization or any other retinal vascular diseases such as retinal vein occlusion documented by FA and (3) eyes with poor en face OCTA images with a signal strength index $<40$ due to media opacity or significant motion artefact. A-scan, B-scan and en face angiography images of both the SCP and DCP were used for analysis.
The en face angiography images were generated by automated layer segmentation of the OCT instrument software. If the automated layer segmentation was inaccurate, manual segmentation was performed to segment the SCP (from $3 \mu \mathrm{m}$ below the internal limiting membrane to $15 \mu \mathrm{m}$ below the inner plexiform layer) and DCP (from $15 \mu \mathrm{m}$ below the inner plexiform layer to $70 \mu \mathrm{m}$ below the inner plexiform layer). This study followed the tenets of the Declaration of Helsinki, and was approved by the Institutional Review Board of the National Taiwan University Hospital. Because of the retrospective nature, the requirement of informed consent was waived.

\section{OCT characteristics}

Central retinal thickness (CRT) and the macular volume were recorded from a $-3 \times 3 \mathrm{~mm}^{2}$ area on the B-scan maps. The presence of ellipsoid zone disruption was defined as any disruption of the integrity of the ellipsoid zone within the $1500-\mu \mathrm{m}$ diameter of fovea in the vertical or horizontal A-scan images.

\section{Image processing}

Image J software version 1.50 (National Institutes of Health, Bethesda, MD, USA) was used to process the en face angiography maps of the SCP and DCP in order to calculate the microvascular parameters. First, the $3 \times 3-\mathrm{mm}$ en face angiography maps were converted to 8 -bit $304 \times 304$-pixel images. Global thresholding was done by subtracting the mean signal of a fixed central selection area (50 pixels) to decrease the background noise. Auto-thresholding was done to adjust the contrast. Two morphological filters (Hessian filter and top-hat filter) were combined and applied to all microvascular images. Afterwards, the images were converted to binary images as described previously by Reif et al. [17]. The skeletonised image was then created by deleting the pixels in the outer boundary of the vessel to 1 pixel (Fig. 1).

\section{Microvascular parameters}

Microvascular parameters, including the numbers of microaneurysms, area of the foveal avascular zone (FAZ), acircularity index of the FAZ, vessel density, skeleton density, vessel density index and fractal dimension were recorded. Microaneurysm was defined as a nodular or comma-shaped structure that was clearly visualised on the OCTA en face images. The area and perimeter of the FAZ were assessed in all images by 1 observer $(\mathrm{C}-\mathrm{C} \mathrm{H})$. The acircularity index of the FAZ was defined as the ratio of the real FAZ perimeter to the best-fit circle perimeter [18, 19]. Vessel density was calculated as the ratio of pixels occupied by blood vessels (white pixels in the binarized image) to all 
(A) Original

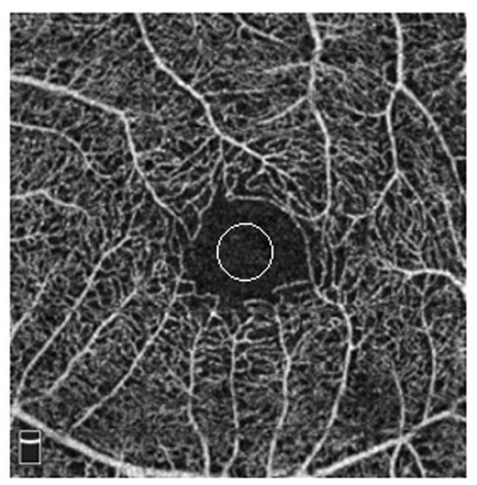

(B) Global thresholding

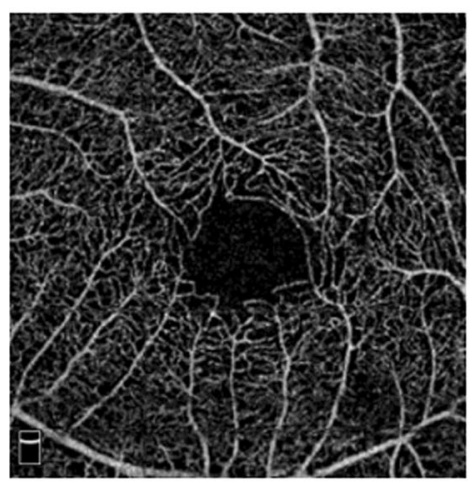

(D) Hessian filter $\square$

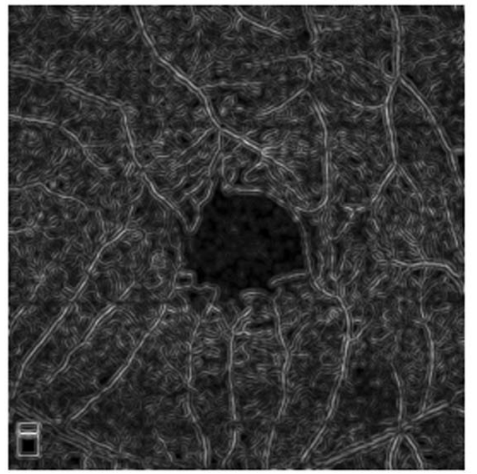

(C) Top-hat filter

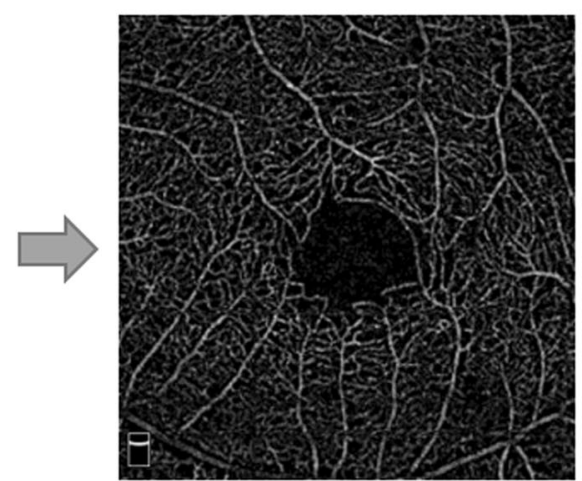

(E) Combination

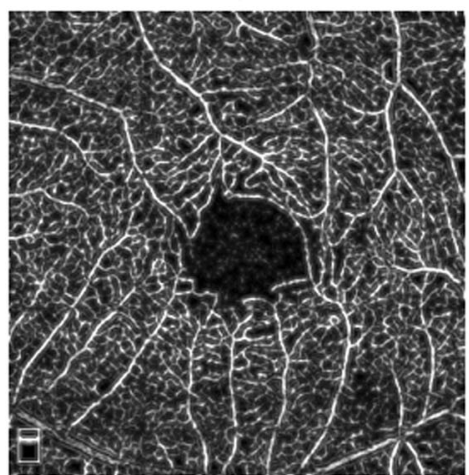

(G) Skeletonization

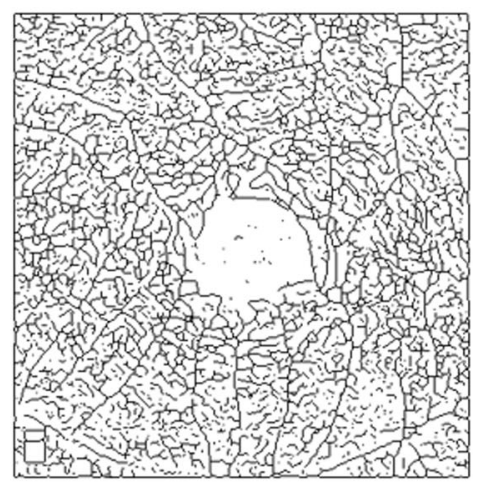

(F) Binarization

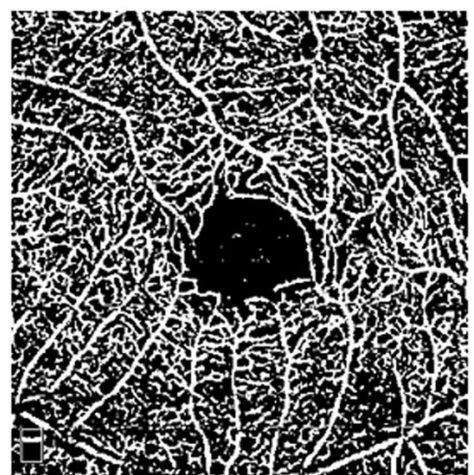

Fig. 1 Imaging processing of a representative case using ImageJ software. a The original en face angiography map of the superficial retinal layer. The white circle demonstrates the manually selected area that was used for global thresholding. b Global thresholding was done by subtracting the mean of the fixed central selection area (50 pixels) to decrease the background noise. c The top-hat filtered image. d The
Hessian filtered image. e Image with the filters combined. f After binarization, the binarized image was used for the quantification of vessel density. $\mathbf{g}$ A skeletonised image was obtained by iterative deletion of the pixels in the outer boundary of the binarized image until only 1 pixel remained along the width direction of the vessels. This final image was used for calculation of skeleton density and fractal dimension pixels in a binarized image. Skeleton density was then calculated as the ratio of pixels occupied by skeletonised vessels to all pixels in a skeletonised image. Vessel diameter index was defined as the ratio of vessel density to skeletonised density. Fractal dimension was determined from a skeletonised image using the box counting method in ImageJ Fiji software.

\section{Statistical analysis}

BCVA was converted into the logarithm of the minimum angle of resolution $(\log M A R)$ for statistical analysis. Due to the correlations between two eyes of the same patients, nonparametric Spearman correlation was used to evaluate the correlations between BCVA and CRT/macular volume, and 
the correlation coefficients $(\rho)$ were calculated. Spearman correlation was also used to assess the correlations between CRT and microvascular parameters. To evaluate the impact of microvascular parameters on BCVA, generalised estimating equation models were used with the patient set as a random effect in order to capture the correlations between two eyes of the same patients. CRT and the presence of ellipsoid zone disruption were also adjusted in the models. A $P$-value of $<0.05$ was considered statistically significant. SAS 9.4 software (SAS Institute Inc., Cary, NC, USA) was used for all statistical analyses.

\section{Results}

\section{Patient characteristics}

A total of 81 eyes from 48 patients were included in the analysis. The mean age of the 48 patients was $62.3 \pm 9.5$ years (range: 44-84 years). Twenty-four patients were female, and 24 were male. Of the 81 eyes, 26 (32.1\%) were treatment naive, and 55 (67.9\%) had received anti-VEGF treatment before. Twenty-nine eyes (35.8\%) had moderate nonproliferative diabetic retinopathy (NPDR), 10 (12.3\%) had severe NPDR and 42 (51.9\%) had proliferative diabetic retinopathy (PDR). The mean $\log$ MAR of BCVA was $0.65 \pm$ 0.39 (range: $0.05-1.52$ ). The mean CRT was $359 \pm 114 \mu \mathrm{m}$ (range: $161-784 \mu \mathrm{m}$ ), and the mean macular volume was $8.73 \pm 1.69 \mathrm{~mm}^{2}$ (range: $5.88-17.91 \mathrm{~mm}^{2}$ ). A thicker CRT $(\rho=0.36, P=0.001)$ and a larger macular volume $(\rho=$ $0.59, P<0.001)$ were both significantly correlated with a poorer BCVA. Eleven of the 81 eyes (13.6\%) had ellipsoid zone disruption at the fovea, and the eyes with ellipsoid zone disruption had a significantly poorer BCVA (mean: $1.10 \pm$ 0.35 ) than those without ellipsoid zone disruption (mean: $0.58 \pm 0.35)(P<0.001)$.

\section{Microvascular parameters}

The mean number of microaneurysms was $4.1 \pm 1.8$ in the SCP and $9.8 \pm 3.7$ in the DCP. The mean area of the FAZ was $0.36 \pm 0.16 \mathrm{~mm}^{2}$ in the SCP and $0.66 \pm 0.25 \mathrm{~mm}^{2}$ in the DCP. Detailed microvascular parameters are shown in Table 1. None of the microvascular parameters in the SCP or DCP were correlated with CRT $(P>0.05$ for all).

\section{Correlations between BCVA and microvascular parameters}

Of the microvascular parameters in SCP, a higher acircularity index of FAZ was correlated with poorer BCVA $(P=$ 0.042). After adjustment for CRT and ellipsoid zone disruption at the fovea, however, such correlation was no
Table 1 Microvascular parameters of the 81 eyes

\begin{tabular}{|c|c|c|c|}
\hline & \multirow[b]{2}{*}{ Mean (SD) } & \multicolumn{2}{|c|}{$\begin{array}{l}\text { Spearman correlation } \\
\text { with CRT }\end{array}$} \\
\hline & & Coefficient $\rho$ & $P$-value \\
\hline \multicolumn{4}{|l|}{ Superficial capillary plexus } \\
\hline Number of microaneurysms & $4.1(1.8)$ & 0.06 & 0.58 \\
\hline Area of the FAZ & $0.36(0.16)$ & -0.25 & 0.087 \\
\hline $\begin{array}{l}\text { Acircularity index of } \\
\text { the FAZ }\end{array}$ & $1.34(0.12)$ & -0.05 & 0.64 \\
\hline Vessel density & $35.76(2.77)$ & -0.08 & 0.47 \\
\hline Skeleton density & $14.58(1.52)$ & -0.04 & 0.71 \\
\hline Vessel density index & $2.47(0.19)$ & -0.02 & 0.89 \\
\hline Fractal dimension & $1.68(0.03)$ & 0.03 & 0.76 \\
\hline \multicolumn{4}{|l|}{ Deep capillary plexus } \\
\hline Number of microaneurysms & $9.8(3.7)$ & 0.16 & 0.15 \\
\hline Area of the FAZ & $0.66(0.25)$ & -0.01 & 0.96 \\
\hline $\begin{array}{l}\text { Acircularity index of } \\
\text { the FAZ }\end{array}$ & $1.43(0.15)$ & 0.10 & 0.39 \\
\hline Vessel density & $36.98(3.18)$ & -0.16 & 0.15 \\
\hline Skeleton density & $15.64(1.76)$ & -0.08 & 0.48 \\
\hline Vessel density index & $2.38(0.20)$ & -0.05 & 0.63 \\
\hline Fractal dimension & $1.70(0.04)$ & -0.06 & 0.62 \\
\hline
\end{tabular}

$C R T$ central retinal thickness, FAZ foveal avascular zone

longer significant $(P=0.18)$. None of the other microvascular parameters of the SCP were correlated with BCVA. In the DCP, lower vessel density, lower skeleton density and lower fractal dimension were correlated with poorer BCVA ( $P=0.006,0.01$ and 0.008 , respectively). After adjustment for CRT and ellipsoid zone disruption at the fovea, lower skeleton density and lower fractal dimension remained correlated with poorer BCVA $(P=0.030$ and 0.024 , respectively) (Table 2). Figure 2 shows three representative cases with different patterns of retinal microvasculature.

\section{Discussion}

OCTA is a noninvasive, effective tool to examine different layers of the retinal microvasculature, and it has been used to evaluate various kinds of retinal vascular diseases [7]. Several quantitative parameters have been obtained from OCTA to represent the structure and perfusion status of retinal microvasculature $[10-13,17]$. Previous studies using OCTA has shown that increased area or irregularity of the FAZ, decreased vessel density, decreased skeleton density and decreased fractal dimension of the central macula were associated with worsening of DR [14, 18, 20]. With respect to the relationship between macular ischaemia and visual acuity, it has been reported that decreased vessel density 
Table 2 Correlations between best-corrected visual acuity and microvascular parameters by linear mixed models

\begin{tabular}{|c|c|c|c|c|c|c|}
\hline & \multicolumn{2}{|c|}{ Simple regression } & \multicolumn{2}{|c|}{ Adjustment for CRT } & \multicolumn{2}{|c|}{$\begin{array}{l}\text { Adjustment for CRT } \\
\text { and ellipsoid zone } \\
\text { disruption }\end{array}$} \\
\hline & Coefficient & $P$-value & Coefficient & $P$-value & Coefficient & $P$-value \\
\hline \multicolumn{7}{|l|}{ Superficial capillary plexus } \\
\hline Number of microaneurysms & -0.008 & 0.76 & -0.016 & 0.52 & -0.008 & 0.72 \\
\hline Area of the FAZ & 0.002 & 0.99 & 0.19 & 0.49 & 0.15 & 0.54 \\
\hline Acircularity index of the FAZ & 0.71 & 0.042 & 0.78 & 0.019 & 0.39 & 0.18 \\
\hline Vessel density & -0.021 & 0.20 & -0.023 & 0.15 & -0.010 & 0.46 \\
\hline Skeleton density & -0.035 & 0.24 & -0.039 & 0.16 & -0.030 & 0.21 \\
\hline Vessel density index & 0.21 & 0.34 & 0.22 & 0.31 & 0.23 & 0.22 \\
\hline Fractal dimension & -1.84 & 0.17 & -2.10 & 0.097 & -1.47 & 0.18 \\
\hline \multicolumn{7}{|l|}{ Deep capillary plexus } \\
\hline Number of microaneurysms & 0.019 & 0.11 & 0.014 & 0.22 & 0.019 & 0.055 \\
\hline Area of the FAZ & -0.068 & 0.69 & -0.14 & 0.41 & -0.043 & 0.76 \\
\hline Acircularity index of the FAZ & 0.50 & 0.086 & 0.42 & 0.13 & 0.22 & 0.35 \\
\hline Vessel density & -0.041 & 0.006 & -0.037 & 0.007 & -0.021 & 0.10 \\
\hline Skeleton density & -0.065 & 0.01 & -0.065 & 0.008 & -0.046 & 0.030 \\
\hline Vessel density index & 0.21 & 0.30 & 0.25 & 0.21 & 0.27 & 0.11 \\
\hline Fractal dimension & -3.23 & 0.008 & -3.27 & 0.005 & -2.30 & 0.024 \\
\hline
\end{tabular}

$C R T$ central retinal thickness, $F A Z$ foveal avascular zone and increased area of the FAZ were correlated with poor vision in patients with DR in the absence of DMO [21]. As for patients with DMO, macular ischaemia in addition to macular oedema and photoreceptor disruption could theoretically also contribute to vision loss. However, the impact of macular ischaemia on visual acuity has not been wellstudied in DMO.

In this study, correlations between visual acuity and microvascular parameters obtained by OCTA, including the numbers of microaneurysms, area of the FAZ, acircularity index of the FAZ, vessel density, skeleton density, vessel density index and fractal dimension of the central macula in the SCP and DCP, were examined in patients with DMO. CRT and ellipsoid zone disruption at the fovea were adjusted in the regression model in order to determine the actual relationship between visual acuity and macular ischaemia. We found that, after adjusting for CRT and ellipsoid zone disruption at the fovea, poor vision in DMO was correlated with low skeleton density and low fractal dimension in the DCP, but not those in the SCP.

Vessel density represents the proportion of vascularises area in the central macula. Low vessel density may result from parafoveal capillary dropout or decreased perfusion, and would theoretically affect vision. On the other hand, skeleton density accounts for only the vascular skeleton network; therefore, low skeleton density is more related to capillary dropout. Fractal dimension represents the branching complexity of visible vessels; that is, dropout of small capillaries will result in a decrease of fractal dimension. The fractal dimension of large retinal vessels measured with colour fundus photography has been shown to have diverse associations with DR and its progression [22-25]. Fractal dimension of the microvasculature measured with OCTA has been shown to decrease in various stages of DR, and the change is more prominent in the DCP than in the SCP [26, 27], possibly due to capillary dropout in the central macula. A recent study showed that the fractal dimensions of the SCP and DCP were higher in patients with DR than in those without DR [28]. In this study, we found that low vessel density, low skeleton density and low fractal dimension in the DCP were all independent factors correlated with poor visual acuity in DMO. This finding is in line with previous reports that capillary dropout in the central macula results in decreased skeleton density, as well as decreased fractal dimension. Poor perfusion will also result in decreased vessel density. Both may contribute to insufficient blood supply in the macula, and result in poor macular function.

The correlations between visual acuity and skeleton density/fractal dimension in the DCP sustained after adjustment for CRT and ellipsoid zone disruption suggest that, in addition to macular oedema and photoreceptor disruption, macular ischaemia with capillary dropout plays an important role in the development of visual impairment in DMO. As for vessel density, the correlation with vision was not significant after adjusted for CRT and ellipsoid zone disruption. It is possible that eyes with capillary dropout have compensatory vessel dilatation as 

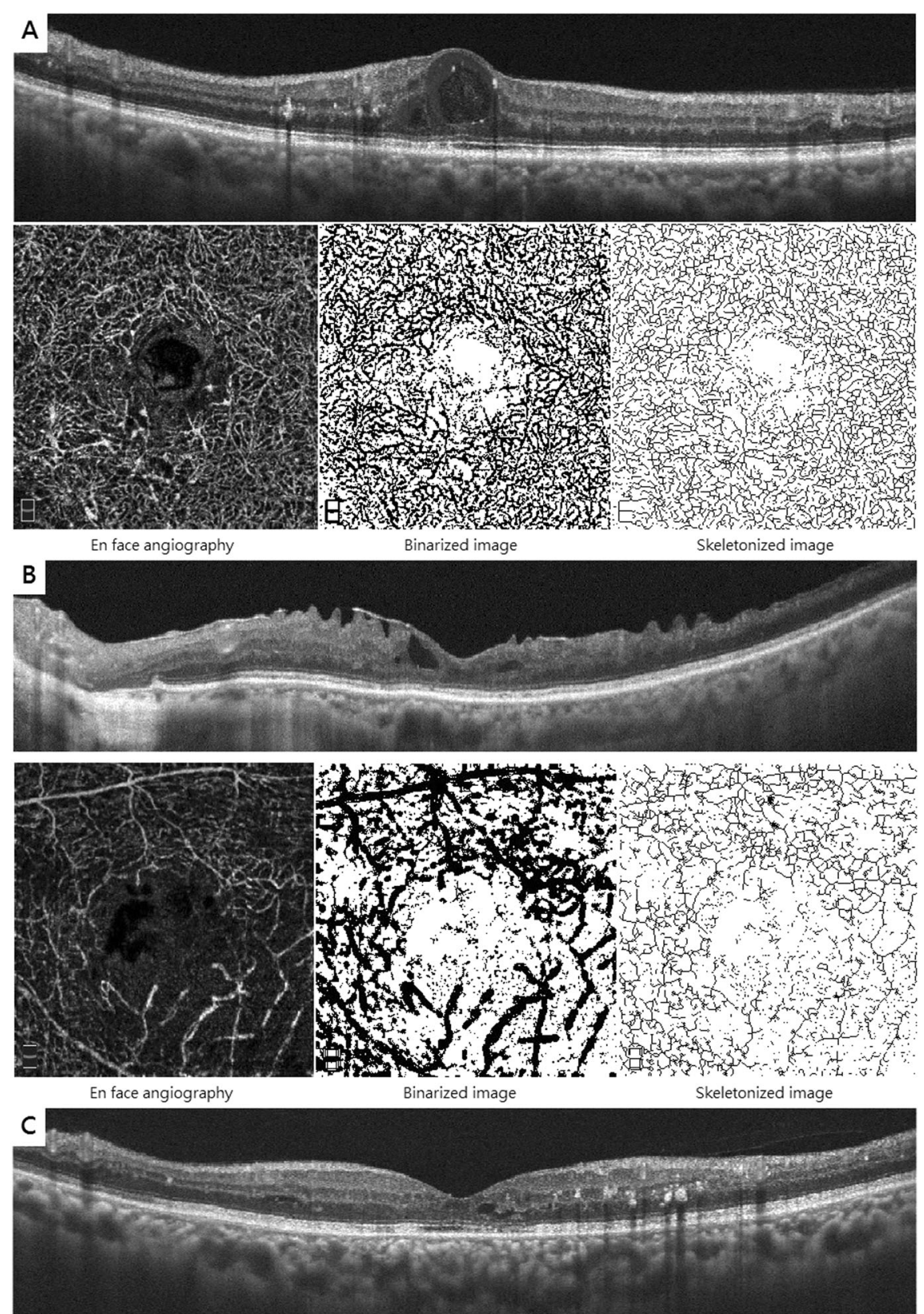

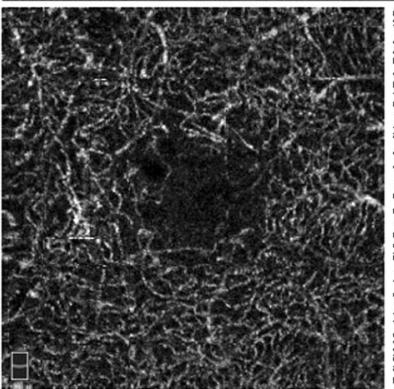

En face angiography

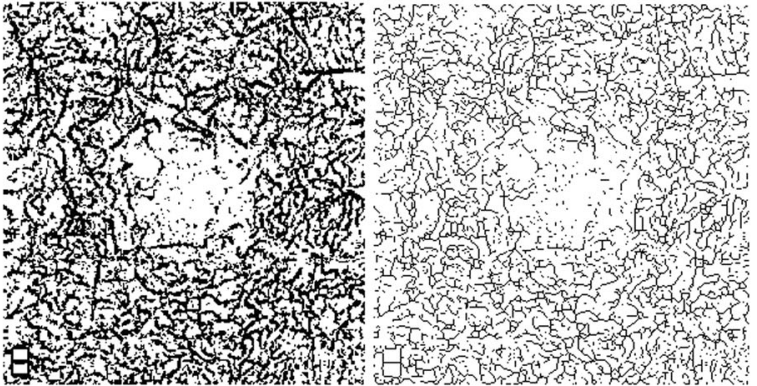

Binarized image

Skeletonized image shown in Fig. 2b, which makes the effect of reduced vessel density less prominent than decreased skeleton density. Based on our results, suboptimal visual improvement after treatment for DMO may be anticipated in patients with low skeleton density or low fractal dimension in the DCP. However, it is not clear if the 
Fig. 2 a Case 1: The OCT B-scan of the right phakic eye of a 68-yearold male showed typical DMO with cystoid oedema at fovea, and hyperreflective foci representing hard exudates at parafoveal area. The en face angiography of DCP showed some juxtafoveal capillary dropout with relative preservation of capillary network at the parafoveal area. The vessel density, skeletal density and fractal dimension were $42.27,18.06$ and 1.74 , respectively. The BCVA was 20/50 at that time and improved to $20 / 25$ after treatment with ranibizumab. b Case 2: The left pseudophakic eye of a 55-year-old male had received 3-monthly injections of ranibizumab for diabetic macular oedema. The OCT B-scan showed some residual intraretinal cysts at the fovea and epiretinal membrane, and retinal folding at the parafoveal area. Besides, the en face angiography of DCP showed severe parafoveal capillary dropout with dilatation of the remaining arterioles. Although the vessel density was not low (41.74), the skeleton density and fractal dimension were both quite low (12.30 and 1.61, respectively). Even though the ellipsoid zone at the fovea was well preserved, the BCVA was only 20/125. c Case 3: The OCT B-scan of the right phakic eye of a 60 -year-old male showed only mild macular oedema with intraretinal cysts at the juxtafoveal area and hyperreflective foci representing hard exudates at parafoveal area. The parafoveal capillary network of DCP looked not as bad as that of Case 2 in the en face angiography. However, we can see the density was actually lower when compared with Case 1 . The vessel density, skeletal density and fractal dimension were all very low $(30.59,13.14$ and 1.63 , respectively). The BCVA was 20/63 and only improved to $20 / 50$ after treatment with ranibizumab

effects of these parameters will change after treatment for DMO. This issue warrants further study.

The current study found no significant associations of microvascular parameters in the SCP with visual acuity. This is compatible with previous studies that have found the DCP was affected more significantly than the SCP in patients with DR or DMO [26, 27, 29]. By contrast, Durbin et al. [30] found that skeleton density in the SCP rather than the DCP was correlated with BCVA in DR. This may be related to the different segmentation methods in different machines. As the segmentation of retinal layers is getting more and more precise in OCTA, further studies may explore this issue with a better resolution in the future.

Balaratnasingam et al. [16] showed that the area of the FAZ was significantly correlated with visual acuity in DR and retinal vein occlusion after adjustment for ellipsoid zone disruption. However, in their study, only 38 of the 65 eyes had macular oedema, and the extent of macular oedema was not adjusted in the analysis. In this study, we found that the area of the FAZ was not correlated with BCVA in DMO. Because the size of the FAZ varies a lot among normal eyes [31], it may not represent the extent of macular ischaemia in a cross-sectional study. On the other hand, a higher acircularity index of the FAZ may be more representative of macular ischaemia than a larger FAZ. Indeed, in this study, we found that a higher acircularity index of the FAZ in SCP was associated with poorer BCVA. Such a correlation became insignificant after adjusted for CRT and ellipsoid zone disruption; further study will be needed to elucidate the relationship.
Hasegawa et al. [32] recently reported that a significantly higher density of microaneurysms is noted in the DCP compared with the SCP. In this study, we also found that the number of microaneurysms was larger in the DCP (mean: 9.8) than in the SCP (mean: 4.1). However, these numbers were not correlated with visual acuity. It is plausible because microaneurysms may not affect vision directly if they do not result in macular oedema.

There are some limitations to this study. First, this was a retrospective cross-sectional study. Therefore, the effect of anti-VEGF treatment on these microvascular parameters and the longitudinal changes could not be evaluated. Confounding factors such as cataracts, severe macular oedema and other media opacity might reduce the detectability of blood flow in retinal vasculature and affect the calculation of vessel density as well as other parameters. Motion artefacts and reproducibility have also been critical issues in OCTA. Therefore, en face images with a strength index $<40$ were excluded from this study. CRT was added in the regression model to adjust for its impact on visual acuity and the calculation of microvascular parameters. Second, severe macular oedema would result in disorganisation of the retinal layers, which make automatic segmentation of the slabs more unreliable. Although manual identification of the slabs was performed, it could not be sure that the segmentation was totally accurate. Furthermore, the quantification of the number of microaneurysms and the area of the FAZ may be affected by subjective judgement. For these reasons, all image processing was performed by the same person to reduce the inter-rater bias. Finally, there was still a problem of projection artefact for the OCTA imaging. However, it was a non-informational bias, and should only affect the power for statistical testing. We expect progress in the hardware and software of OCTA would improve this problem in the future.

In conclusion, we found that low skeleton density and low fractal dimension in the DCP measured with OCTA were significantly correlated with poor vision in DMO after adjustment for CRT and ellipsoid zone disruption at the fovea. These results demonstrate that macular ischaemia with capillary dropout plays an important role in the development of visual disturbance in DMO, and OCTA could offer quantified parameters of macular microvasculature to measure its impact on visual acuity.

\section{Summary}

\section{What was known before}

- As diabetic retinopathy worsens, the vessel density, skeleton density and branching complexity of macular 
microvasculature measured with optical coherence tomography angiography (OCTA) all decreased.

- Eyes with diabetic macular oedema (DME) may also have concurrent macular ischaemia.

\section{What this study adds}

- Lower skeleton density and lower branching complexity in the deep capillary plexus of macula measured with OCTA were correlated with poorer visual acuity in eyes with DME after adjustment for central retinal thickness and ellipsoid zone disruption at the fovea.

- Macular ischaemia plays an important role in the development of visual disturbance in DME, and OCTA could offer quantified parameters of macular microvasculature to show its impact on visual acuity.

\section{Compliance with ethical standards}

Conflict of interest The authors declare that they have no conflict of interest.

Publisher's note: Springer Nature remains neutral with regard to jurisdictional claims in published maps and institutional affiliations.

\section{References}

1. Yau JW, Rogers SL, Kawasaki R, Lamoureux EL, Kowalski JW, Bek T, et al. Global prevalence and major risk factors of diabetic retinopathy. Diabetes care. 2012;35:556-64.

2. Funatsu $H$, Yamashita $H$, Noma $H$, Mimura $T$, Yamashita $T$, Hori S. Increased levels of vascular endothelial growth factor and interleukin-6 in the aqueous humor of diabetics with macular edema. Am J Ophthalmol. 2002;133:70-7.

3. Bhagat N, Grigorian RA, Tutela A, Zarbin MA. Diabetic macular edema: pathogenesis and treatment. Surv Ophthalmol. 2009;54:1-32.

4. Spaide RF, Klancnik JM Jr., Cooney MJ. Retinal vascular layers imaged by fluorescein angiography and optical coherence tomography angiography. JAMA Ophthalmol. 2015;133:45-50.

5. Lee J, Rosen R. Optical Coherence Tomography Angiography in Diabetes. Curr Diab Rep. 2016;16:123.

6. Tan ACS, Tan GS, Denniston AK, Keane PA, Ang M, Milea D, et al. An overview of the clinical applications of optical coherence tomography angiography. Eye. 2018;32:262-86.

7. Kashani AH, Chen CL, Gahm JK, Zheng F, Richter GM, Rosenfeld PJ, et al. Optical coherence tomography angiography: a comprehensive review of current methods and clinical applications. Prog Retin Eye Res. 2017;60:66-100.

8. Jia Y, Tan O, Tokayer J, Potsaid B, Wang Y, Liu JJ, et al. Splitspectrum amplitude-decorrelation angiography with optical coherence tomography. Opt Express. 2012;20:4710-25.

9. Bonnin S, Mane V, Couturier A, Julien M, Paques M, Tadayoni $\mathrm{R}$, et al. New insight into the macular deep vascular plexus imaged by optical coherence tomography angiography. Retina. 2015;35:2347-52.
10. Hwang TS, Jia Y, Gao SS, Bailey ST, Lauer AK, Flaxel CJ, et al. Optical coherence tomography angiography features of diabetic retinopathy. Retina. 2015;35:2371-6.

11. Ristanovic D, Stefanovic BD, Puskas N. Fractal analysis of dendrite morphology using modified box-counting method. Neurosci Res. 2014;84:64-7.

12. Li TG, Wang S, Zhao N. Fractal research of pathological tissue images. Comput Med Imaging Graph. 2007;31:665-71.

13. Chu Z, Lin J, Gao C, Xin C, Zhang Q, Chen CL, et al. Quantitative assessment of the retinal microvasculature using optical coherence tomography angiography. J Biomed Opt. 2016;21:66008.

14. Kim AY, Chu Z, Shahidzadeh A, Wang RK, Puliafito CA, Kashani AH. Quantifying microvascular density and morphology in diabetic retinopathy using spectral-domain optical coherence tomography angiography. Invest Ophthalmol Vis Sci. 2016;57:362-70.

15. Coscas G, Lupidi M, Coscas F. Optical coherence tomography angiography in diabetic maculopathy. Dev Ophthalmol. 2017; 60:38-49.

16. Balaratnasingam $\mathrm{C}$, Inoue $\mathrm{M}$, Ahn S, McCann J, Dhrami-Gavazi E, Yannuzzi LA, et al. Visual acuity is correlated with the area of the foveal avascular zone in diabetic retinopathy and retinal vein occlusion. Ophthalmology. 2016;123:2352-67.

17. Reif R, Qin J, An L, Zhi Z, Dziennis S, Wang R. Quantifying optical microangiography images obtained from a spectral domain optical coherence tomography system. Int $\mathrm{J}$ Biomed Imaging. 2012;2012:509783.

18. Krawitz BD, Mo S, Geyman LS, Agemy SA, Scripsema NK, Garcia PM, et al. Acircularity index and axis ratio of the foveal avascular zone in diabetic eyes and healthy controls measured by optical coherence tomography angiography. Vis Res. 2017; 139:177-86.

19. Tam J, Dhamdhere KP, Tiruveedhula P, Manzanera S, Barez S, Bearse MA Jr., et al. Disruption of the retinal parafoveal capillary network in type 2 diabetes before the onset of diabetic retinopathy. Invest Ophthalmol Vis Sci. 2011;52:9257-66.

20. Freiberg FJ, Pfau M, Wons J, Wirth MA, Becker MD, Michels S. Optical coherence tomography angiography of the foveal avascular zone in diabetic retinopathy. Graefes Arch Clin Exp Ophthalmol. 2016;254:1051-8.

21. Samara WA, Shahlaee A, Adam MK, Khan MA, Chiang A, Maguire JI, et al. Quantification of diabetic macular ischemia using optical coherence tomography angiography and its relationship with visual acuity. Ophthalmology. 2017;124:235-44.

22. Broe R, Rasmussen ML, Frydkjaer-Olsen U, Olsen BS, Mortensen HB, Peto T, et al. Retinal vascular fractals predict longterm microvascular complications in type 1 diabetes mellitus: the Danish Cohort of Pediatric Diabetes 1987 (DCPD1987). Diabetologia. 2014;57:2215-21.

23. Cheung N, Donaghue KC, Liew G, Rogers SL, Wang JJ, Lim $\mathrm{SW}$, et al. Quantitative assessment of early diabetic retinopathy using fractal analysis. Diabetes Care. 2009;32:106-10.

24. Lim LS, Chee ML, Cheung CY, Wong TY. Retinal vessel geometry and the incidence and progression of diabetic retinopathy. Invest Ophthalmol Vis Sci. 2017;58:BIO200-BIO205.

25. Talu S, Calugaru DM, Lupascu CA. Characterisation of human non-proliferative diabetic retinopathy using the fractal analysis. Int J Ophthalmol. 2015;8:770-6.

26. Bhardwaj S, Tsui E, Zahid S, Young E, Mehta N, Agemy S, et al. Value of fractal analysis of optical coherence tomography angiography in various stages of diabetic retinopathy. Retina. 2018;38:1816-23.

27. Chen Q, Ma Q, Wu C, Tan F, Chen F, Wu Q, et al. Macular vascular fractal dimension in the deep capillary layer as an early 
indicator of microvascular loss for retinopathy in type 2 diabetic patients. Invest Ophthalmol Vis Sci. 2017;58:3785-94.

28. Ting DSW, Tan GSW, Agrawal R, Yanagi Y, Sie NM, Wong CW, et al. Optical coherence tomographic angiography in type 2 diabetes and diabetic retinopathy. JAMA Ophthalmol. 2017;135:306-12.

29. Lee J, Moon BG, Cho AR, Yoon YH. Optical Coherence tomography angiography of DME and its association with Anti-VEGF treatment response. Ophthalmology. 2016;123:2368-75.

30. Durbin MK, An L, Shemonski ND, Soares M, Santos T, Lopes M, et al. Quantification of retinal microvascular density in optical coherence tomographic angiography images in diabetic retinopathy. JAMA Ophthalmol. 2017;135:370-6.

31. Samara WA, Say EA, Khoo CT, Higgins TP, Magrath G, Ferenczy S, et al. Correlation of foveal avascular zone size with foveal morphology in normal eyes using optical coherence tomography angiography. Retina. 2015;35:2188-95.

32. Hasegawa N, Nozaki M, Takase N, Yoshida M, Ogura Y. New insights into microaneurysms in the deep capillary plexus detected by optical coherence tomography angiography in diabetic macular edema. Invest Ophthalmol Vis Sci. 2016;57:348-55. 